\title{
Autocorrelation and Linear Complexity of Binary Generalized Cyclotomic Sequences with Period pq
}

\author{
Yan Wang, Liantao Yan (iD, Qing Tian, and Liping Ding \\ School of Science, Xi'an University of Architecture and Technology, Xi'an 710055, China \\ Correspondence should be addressed to Liantao Yan; yanliantao@xauat.edu.cn
}

Received 26 February 2021; Revised 27 March 2021; Accepted 3 April 2021; Published 26 April 2021

Academic Editor: Tianping Zhang

Copyright (c) 2021 Yan Wang et al. This is an open access article distributed under the Creative Commons Attribution License, which permits unrestricted use, distribution, and reproduction in any medium, provided the original work is properly cited.

Ding constructed a new cyclotomic class $\left(V_{0}, V_{1}\right)$. Based on it, a construction of generalized cyclotomic binary sequences with period $p q$ is described, and their autocorrelation value, linear complexity, and minimal polynomial are confirmed. The autocorrelation function $C_{S}(w)$ is 3-level if $p \equiv 3 \bmod 4$, and $C_{S}(w)$ is 5-level if $p \equiv 1 \bmod 4$. The linear complexity $\operatorname{LC}(S)>(p q / 2)$ if $p \equiv 1 \bmod 8, p>q+1$, or $p \equiv 3 \bmod 4$ or $p \equiv-3 \bmod 8$. The results show that these sequences have quite good cryptographic properties in the aspect of autocorrelation and linear complexity.

\section{Introduction}

Pseudorandom sequences with good cryptography properties have wide applications in CDMA, global positing systems, and stream ciphers. The security of stream ciphers depends on the randomness of the key stream, which makes the construction of pseudorandom sequences to be an important research direction. Many researchers focused on cyclotomic sequences, which have good balance property. Linear complexity and autocorrelation are important criteria for measuring unpredictability of cyclotomic sequences.

Let $F_{l}$ denote a finite field with $l$ elements, where $l$ is a prime power. A sequence $S=\left\{s_{i}\right\}$ is periodic if there exists a positive integer $N$ such that $s_{j+N}=s_{j}$ for all $j \geq 0$.

Let $S=\left\{s_{i}\right\}$ be a periodic sequence over $F_{l}$ with period $N$. The periodic autocorrelation function of binary sequence $S$ is defined by

$$
C_{S}(w)=\sum_{i=0}^{N-1}(-1)^{s_{i+w}+s_{i}}
$$

where $0 \leq w \leq N-1$.

Autocorrelation function measures the amount of similarity between sequence $S$ and a shift of $S$ by $w$ shifts. Only when the values of $C_{S}(w)$ distribute flat and low, sequence $S$ is easy to distinguish from each time shifted version of itself. The autocorrelation function with the ideal distribution of values is two-valued, which is given as

$$
C_{S}(w)= \begin{cases}N & \text { if } w=0, \\ 1, & \text { otherwise }\end{cases}
$$

Sequences with ideal autocorrelation functions have many applications in cryptography, coding, and other communication engineering.

Linear complexity of $S$, denoted by $\operatorname{LC}(S)$, is the least integer $L$ of a linear recurrence relation over $F_{l}$ satisfied by $S$ :

$$
-c_{0} s_{i+L}=c_{1} s_{i+L-1}+\cdots+c_{L} s_{i}, \quad i \geq 0,
$$

where $c_{0}, c_{1}, \ldots, c_{L} \in F_{l}$. The linear complexity of a sequence is also defined to be the length of the shortest linear feedback shift register which can generate the sequence. It is an important criterion of randomness of sequences in stream ciphers. To resist the attack from Berlekamp-Massey algorithm, the sequences used in cipher systems should have large linear complexity. If $\mathrm{LC}(S) \geq(N / 2)$, where $N$ is the least period of $S$, then $S$ is considered to be good from the viewpoint of linear complexity.

The minimal polynomial $m(x)$ of $S$ is 


$$
m(x)=\frac{x^{N}-1}{\operatorname{gcd}\left(x^{N}-1, S(x)\right)},
$$

and the linear complexity $\operatorname{LC}(S)$ of $S$ is given by $N-\operatorname{deg}\left(\operatorname{gcd}\left(x^{N}-1, S(x)\right)\right)$, where $S(x)$ is the generating polynomial of $S$, that is,

$$
S(x)=s_{0}+s_{1} x+s_{2} x^{2}+\cdots+s_{N-1} x^{N-1} .
$$

Sequences from cyclotomic and generalized cyclotomic are important families of pseudorandom sequences.

For an integer $N \geq 2$, let $Z_{N}=\{0,1, \ldots, N-1\}$ denote the residue class ring of integers modulo $N$ and $Z_{N}^{*}$ be the multiplicative group consisting of all invertible elements in $Z_{N}$. A partition $\left\{D_{0}^{(N)}, D_{1}^{(N)}, \ldots, D_{d-1}^{(N)}\right\}$ of $Z_{N}^{*}$ is a family of sets satisfying $D_{i}^{(N)} \cap D_{j}^{(N)}=\varnothing$ for all $i \neq j$ and $Z_{N}^{*}=\cup_{i=0}^{d-1} D_{i}^{(N)}$. Suppose $D_{0}^{(N)}$ is a multiplicative subgroup of $Z_{N}^{*}$ and there exist elements $g \in Z_{N}^{*}$ such that $D_{i}^{(N)}=$ $g^{i} D_{0}^{(N)}$ for all $i=1, \ldots, d-1$; then, $D_{i}^{(N)}$ are called classical cyclotomic classes of order $d$ with respect to $N$ when $N$ is prime and generalized cyclotomic classes of order $d$ with respect to $N$ when $N$ is composite. The sequences constructed by them are called classical cyclotomic sequences and generalized cyclotomic sequences, respectively. Gauss [1] first proposed the concept of cyclotomic, divided the multiplicative group $Z_{p}^{*}$, and then divided the residual class ring $Z_{p}$ to construct Gauss classical cyclotomic. Whiteman [2] divided the multiplicative group $Z_{p q}^{*}$ and then divided the residual class ring $Z_{p q}$ to construct Whiteman generalized cyclotomic. Ding and Helleseth [3] divided the multiplicative group $Z_{p_{1}^{e_{1}} p_{2}^{e_{2}} \ldots p_{t}^{e_{t}}}^{e}$ and then divided the residual class ring $Z_{p_{1}^{e_{1}} p_{2}^{e_{2}} \ldots p_{t}^{e_{t}}}$ to construct the Ding generalized cyclotomic. The above three kinds of cyclotomic theories are the most representative and the most widely used cyclotomic theories.

Classical cyclotomic sequences include Legendre sequences, $d$-degree residual sequences, and Hall sextic residue sequences. Damgaard [4] determined the autocorrelation value of Legendre sequences, and then Ding et al. [5] determined their linear complexity. Kim and Song [6] determined the linear complexity of Hall sextic residue sequences.

Ding [7] constructed Whiteman generalized cyclotomic sequences of order 2 and confirmed their linear complexity. And Ding [8] determined the autocorrelation value of Whiteman generalized cyclotomic sequences of order 2. Bai [9] constructed Whiteman generalized cyclotomic sequences of order 4 and determined their linear complexity. Yan et al. [10] extended Whiteman generalized cyclotomic sequences to the case of order $2^{k}$.

Bai [9] determined the autocorrelation value of Ding generalized cyclotomic sequences with period $p q$ of order 2 . And Bai et al. [11] confirmed they had high linear complexity. Yan et al. [12] constructed Ding generalized cyclotomic sequences with period $p^{m}$ and confirmed they had high linear complexity. Edemskiy [13] constructed a kind of balanced binary generalized cyclotomic sequences with period $p^{n+1}$. Zhang et al. [14] determined the linear complexity of generalized cyclotomic sequences with period $2 p^{m}$. Hu et al. [15] constructed generalized cyclotomic sequences with period $p^{m+1} q^{n+1}$ and determined their linear complexity. Ke et al. [16] determined the linear complexity and the autocorrelation value of Ding generalized cyclotomic sequences with period $2 p^{m}$. Chang et al. [17] constructed binary generalized cyclotomic sequences with period $p q r$ and determined their linear complexity and minimal polynomial.

Ding [18] constructed a new cyclotomic class $\left(V_{0}, V_{1}\right)$ and obtained a kind of cyclic code from it. Liu and Chen [19] determined binary generalized cyclotomic sequences with period $p q$ based on the new cyclotomic class $\left(V_{0}, V_{1}\right)$ and determined their autocorrelation value, linear complexity, and minimal polynomial.

In this paper, based on Ding's new cyclotomic class $\left(V_{0}, V_{1}\right)$, a simple construction of binary generalized cyclotomic sequences with period $p q$ is constructed, and their autocorrelation value, linear complexity, and minimal polynomial are confirmed. The remainder of this paper is organized as follows. Section 2 proposes a construction of generalized cyclotomic binary sequences. Section $3 \mathrm{calcu}-$ lates the autocorrelation value, linear complexity, and minimal polynomial of the new sequences and compares our results with [19]. Section 4 concludes this paper.

\section{Preliminaries}

Lemma 1 (see [20]). Let $m_{1}>0, m_{2}>0, a_{1}$, and $a_{2}$ be integers. The system of congruences,

$$
\left\{\begin{array}{l}
x \equiv a_{1}\left(\bmod m_{1}\right), \\
x \equiv a_{2}\left(\bmod m_{2}\right),
\end{array}\right.
$$

has solutions if and only if $\operatorname{gcd}\left(m_{1}, m_{2}\right) \mid a_{1}-a_{2}$.

If the above condition is satisfied, the solution is unique modulo $\mathrm{lcm}\left(m_{1}, m_{2}\right)$.

Let $N=p q$, where $p$ and $q$ are two distinct odd primes. Let $g$ be the unique common primitive root of $p$ and $q$. The existence and uniqueness of $g$ are guaranteed by Lemma 1 . Similarly, there exists a unique integer $x$ which satisfies the following system of congruences:

$$
\left\{\begin{array}{l}
x \equiv g(\bmod p) \\
x \equiv 1(\bmod q) .
\end{array}\right.
$$

Let $d=\operatorname{gcd}(p-1, q-1)$ and $e=(\operatorname{gcd}(p-1)(q-1) / d)$. According to Whiteman [2], Whiteman generalized cyclotomic class of order $d$ is

$$
D_{i}=\left\{g^{s} x^{i}: s=0,1, \ldots, e-1\right\}, \quad i=0,1, \ldots, d-1 .
$$

It can be easily seen that $D_{i} \cap D_{j}^{(N)}=\varnothing$ for all $i \neq j$ and $Z_{N}^{*}=\cup_{i=0}^{d-1} D_{i}$.

Define two sets 


$$
\begin{aligned}
& P=\{p, 2 p, \ldots,(q-1) p\} \\
& Q=\{q, 2 q, \ldots,(p-1) q\} .
\end{aligned}
$$

Then,

$$
Z_{p q}=\bigcup_{i=0}^{d-1} D_{i} \cup P \cup Q \cup\{0\}
$$

Lemma 2 (see [7]). Let

$$
\begin{aligned}
d & =\operatorname{gcd}(p-1, q-1)=2, \\
C_{0} & =\{0\} \cup Q \cup D_{0}, \\
C_{1} & =P \cup D_{1} .
\end{aligned}
$$

And a new binary generalized cyclotomic sequence $S$ of order 2 is

$$
s_{i}= \begin{cases}0, & \text { if } i \in C_{0}, \\ 1, & \text { if } i \in C_{1} .\end{cases}
$$

Let $m=\operatorname{ord}_{p q}$ (2) and $\alpha$ be a primitive pqth root of unity in finite field $F_{2^{m}}, d_{i}(x)=\prod_{j \in D_{i}}\left(x-\alpha^{i}\right), i=0,1$. Then,

(1) When $p \equiv 1 \bmod 8, q \equiv 3 \bmod 8$ or $p \equiv-3 \bmod 8$, $q \equiv-1 \bmod 8$,

$$
\begin{aligned}
\mathrm{LC}(S) & =p q-1, \\
m(x) & =\frac{x^{p q}-1}{x-1} .
\end{aligned}
$$

(2) When $p \equiv-1 \bmod 8, q \equiv 3 \bmod 8$ or $p \equiv 3 \bmod 8$, $q \equiv-1 \bmod 8$,

$$
\begin{gathered}
\operatorname{LC}(S)=(p-1) q, \\
m(x)=\frac{x^{p q}-1}{x^{q}-1} .
\end{gathered}
$$

(3) When $p \equiv-1 \bmod 8, q \equiv-3 \bmod 8$ or $p \equiv 3 \bmod 8$, $q \equiv 1 \bmod 8$

$$
\begin{aligned}
& \mathrm{LC}(S)=p q-p-q+1, \\
& m(x)=\frac{\left(x^{p q}-1\right)(x-1)}{\left(x^{p}-1\right)\left(x^{q}-1\right)} .
\end{aligned}
$$

(4) When $p \equiv 1 \bmod 8, q \equiv-1 \bmod 8$ or $p \equiv-3 \bmod 8$, $q \equiv 3 \bmod 8$,

$$
\begin{aligned}
\mathrm{LC}(S) & =\frac{p q+p+q-3}{2} \\
m(x) & =\frac{x^{p q}-1}{(x-1) d_{0}(x)} .
\end{aligned}
$$

(5) When $p \equiv-1 \bmod 8, q \equiv 1 \bmod 8$ or $p \equiv 3 \bmod 8$, $q \equiv-3 \bmod 8$,

$$
\begin{aligned}
\operatorname{LC}(S) & =\frac{(p-1)(q-1)}{2}, \\
m(x) & =d_{1}(x) .
\end{aligned}
$$

(6) When $p \equiv-1 \bmod 8, q \equiv 1 \bmod 8$ or $p \equiv 3 \bmod 8$, $q \equiv 3 \bmod 8$

$$
\begin{aligned}
\operatorname{LC}(S) & =\frac{(p-1)(q+1)}{2}, \\
m(x) & =\frac{\left(x^{p}-1\right) d_{1}(x)}{x-1} .
\end{aligned}
$$

Lemma 3 (see [8]). Let

$$
\begin{aligned}
d & =\operatorname{gcd}(p-1, q-1)=2, \\
C_{0} & =\{0\} \cup Q \cup D_{0}, \\
C_{1} & =P \cup D_{1} .
\end{aligned}
$$

And a binary generalized cyclotomic sequence $S$ of order 2 is defined by

$$
s_{i}= \begin{cases}0, & \text { if } i \in C_{0}, \\ 1, & \text { if } i \in C_{1} .\end{cases}
$$

Then,

(1) When $((p-1)(q-1) / 4)$ is even,

$$
C_{S}(w)= \begin{cases}q-p-3, & w \in P, \\ 1+p-q, & w \in Q, \\ -1, & w \in Z_{N}^{*} .\end{cases}
$$

(2) When $((p-1)(q-1) / 4)$ is odd,

$$
C_{S}(w)= \begin{cases}q-p-3, & w \in P \\ 1+p-q, & w \in Q \\ -3, & w \in D_{0} \\ 1, & w \in D_{1} .\end{cases}
$$

Lemma 4 (see [9]). Let

$$
\begin{aligned}
d & =\operatorname{gcd}(p-1, q-1)=4, \\
C_{0} & =\{0\} \cup Q \cup D_{0} \cup D_{2}, \\
C_{1} & =P \cup D_{1} \cup D_{3} .
\end{aligned}
$$

And a binary generalized cyclotomic sequence $S$ of order 4 is defined by

$$
s_{i}= \begin{cases}0 & \text { if } i \in C_{0} \\ 1 & \text { if } i \in C_{1} .\end{cases}
$$

(1) If $2 \in D_{0}$ or $2 \in D_{2}$, then 


$$
\operatorname{LC}(S)=\frac{(p+1)(q-1)}{2}
$$

If $2 \in D_{1}$ or $2 \in D_{3}$, then

$$
\mathrm{LC}(S)=p(q-1) \text {. }
$$

(2) When $p \equiv-1 \bmod 8, q \equiv 3 \bmod 8$ or $p \equiv 3 \bmod 8$, $q \equiv-1 \bmod 8$,

$$
C_{S}(w)= \begin{cases}p q, & w \in 0, \\ q-p-3, & w \in P, \\ 1+p-q, & w \in Q \\ 1, & w \in D_{0} \cup D_{2}, \\ -3, & w \in D_{1} \cup D_{3} .\end{cases}
$$

Lemma 5 (see [10]). Let

$$
\begin{aligned}
d & =\operatorname{gcd}(p-1, q-1)=2^{k}, \\
C_{0} & =\{0\} \cup Q \cup\left(\bigcup_{i=0}^{2^{k-1}-1} D_{i}\right), \\
C_{1} & =P \cup\left(\bigcup_{i=2^{k-1}}^{\bigcup^{k}-1} D_{i}\right) .
\end{aligned}
$$

And a binary generalized cyclotomic sequence $S$ of order $2^{k}$ is defined by

$$
s_{i}= \begin{cases}0, & \text { if } i \in C_{0}, \\ 1, & \text { if } i \in C_{1} .\end{cases}
$$

Let $m=\operatorname{ord}_{p q}(2)$ and $\alpha$ be a pqth primitive root of unity in finite field $F_{2^{m}}$,

$$
\begin{aligned}
& S_{i}(x)=\sum_{j \in P \cup\left(\begin{array}{c}
n^{2^{k}-1+i} D_{t=2^{k-1}+i} \\
t
\end{array}\right)} x^{j}, \quad i=0,1, \ldots, 2^{k}-1, \\
& \Lambda=\left\{i_{j}: S_{i_{j}}(\alpha)=0, \quad j=0,1, \ldots, 2^{k-1}-1\right\} .
\end{aligned}
$$

Then,

(1) If for all $s, g^{s} \equiv 2 \bmod p q$ is true,

$$
\begin{gathered}
\operatorname{LC}(S)=(p-1) q, \\
m(x)=\frac{x^{p q}-1}{x^{q}-1} .
\end{gathered}
$$

(2) When there exists $s$ such that $g^{s} \equiv 2 \bmod p q$,

$$
\begin{aligned}
\operatorname{LC}(S) & =\frac{(p+1)(q-1)}{2}, \\
m(x) & =\frac{x^{p q}-1}{\left(x^{p}-1\right)\left(\prod_{i \in \Lambda} d_{i}(x)\right)},
\end{aligned}
$$

where $d_{i}(x)=\prod_{j \in D_{i}}\left(x-\alpha^{j}\right)$.

The following are Ding's new cyclotomic class $\left(V_{0}, V_{1}\right)$.
Assume $d=\operatorname{gcd}(p-1, q-1)=2$; let

$$
\begin{array}{ll}
V_{0}=\left\{g^{s} x^{l}: 0 \leq s \leq e-1,\right. & 0 \leq l \leq d-1,2 \mid s+l\}, \\
V_{1}=\left\{g^{s} x^{l}: 0 \leq s \leq e-1,\right. & 0 \leq l \leq d-1,2 \nmid s+l\} .
\end{array}
$$

With the above preparations, a partition of $Z_{N}^{*}$ is

$$
Z_{N}^{*}=V_{0} \cup V_{1} \text {, }
$$

Then,

$$
Z_{p q}=V_{0} \cup V_{1} \cup P \cup Q \cup\{0\}
$$

Let

$$
\begin{aligned}
& C_{0}=\{0\} \cup Q \cup V_{0}, \\
& C_{1}=P \cup V_{1} .
\end{aligned}
$$

And a binary generalized cyclotomic sequence $S$ with period pq constructed in [19] is

$$
s_{i}= \begin{cases}0, & \text { if } i \in C_{0}, \\ 1, & \text { if } i \in C_{1} .\end{cases}
$$

Lemma 6 (see [19]). Let $S=\left\{s_{i}\right\}$ be the binary sequences defined. Then, the autocorrelation of $S$ is

$$
C_{S}(w)= \begin{cases}p q-2 p-2, & w \in P, \\ 1+p-q-(q-1) \eta_{w}\left((-1)^{(p-1 / 2)}+1\right), & w \in Q, \\ -q-(q-2) \eta_{w}\left((-1)^{(p-1 / 2)}+1\right), & w \in Z_{N}^{*} .\end{cases}
$$

Lemma 7 (see [19]). Let $d_{i}(x)=\prod_{j \in V_{i}}\left(x-\alpha^{i}\right), i=0,1$. Then,

(1) When $p \equiv 1 \bmod 8$,

$$
\mathrm{LC}(S)=\frac{p q+q-p-1}{2}
$$

(2) When $p \equiv-1 \bmod 8$,

$$
\mathrm{LC}(S)=\frac{p q-p-q+1}{2} .
$$

(3) When $p \equiv 3 \bmod 8$,

$$
\mathrm{LC}(S)=p q-p-q+1 \text {. }
$$

(4) When $p \equiv-3 \bmod 8$,

$$
\mathrm{LC}(S)=p q-p .
$$

Now, let 
Journal of Mathematics

$$
\begin{aligned}
& C_{0}=\{0\} \cup P \cup V_{0}, \\
& C_{1}=Q \cup V_{1} .
\end{aligned}
$$

A new binary cyclotomic sequence $S=\left\{s_{i}\right\}$ with period $p q$ is defined by

$$
s_{i}= \begin{cases}0 & \text { if }(i \bmod p q) \in C_{0}, \\ 1 & \text { if }(i \bmod p q) \in C_{1} .\end{cases}
$$

\section{Main Results}

3.1. Autocorrelation of Our New Sequences. Let $\eta_{i}= \begin{cases}1 & \text { if } i \text { is the quadratic residue of module } p \\ -1 & \text { if } i \text { is not the quadratic residue of module } p .\end{cases}$

Lemma 8 (see [19]). Let $V_{0}$ and $V_{1}$ be the sets defined above; then,

(1) $2 \in V_{0}$ if and only if $p \equiv \pm 1(\bmod 8)$

(2) $2 \in V_{1}$ if and only if $p \equiv \pm 3(\bmod 8)$

Lemma 9 (see [19]). Let $V_{0}$ be the sets defined above; then, $n \in V_{0}$ if and only if $(1 / 2)\left(1+\eta_{n}\right)=1,0 \leq n \leq N-1$.

Lemma 10. Let $1 \leq w \leq N-1$; then,
(1) $\sum_{\substack{\operatorname{gcd}(i, p q)=1 \\ \operatorname{gcd}(i+w, p q)=1}}^{N-1} \eta_{i(i+w)}= \begin{cases}(q-2)(p-1), & w \in P, \\ 1-q, & w \in Q, \\ 2-q, & w \in Z_{N}^{*}\end{cases}$

(2) $\sum_{\substack{\operatorname{gdc}(i, p q)=1 \\ p(i+w)}}^{N-1} \eta_{i}= \begin{cases}0, & w \in P, \\ (q-1) \eta_{-w}, & w \notin P .\end{cases}$

(3) $\sum_{\substack{i=0 \\ \operatorname{gcd}(i+w, p q)=1}}^{N-1} \eta_{i+w}= \begin{cases}0, & w \in P, \\ (q-1) \eta_{w}, & w \notin P .\end{cases}$

(4) $\underset{\substack{\operatorname{gc}(=0)=1 \\ \text { qli }(i, w) p q)+i+w}}{N-1} \eta_{i}= \begin{cases}-\eta_{-w} & w \in Z_{N}^{*}, \\ 0 & w \notin Z_{N}^{*},\end{cases}$

(5) $\sum_{\substack{i=1 \\ \operatorname{gcd}(i+w, p q)=1}}^{N-1} \eta_{i+w}= \begin{cases}-\eta_{w} & w \in Z_{N}^{*}, \\ 0 & w \notin Z_{N}^{*}\end{cases}$

(6) $\sum_{\substack{\text { i=0 } \\ p l i \\ p \mid i+w}}^{N-1} 1= \begin{cases}q, & w \in P, \\ 0, & w \notin P .\end{cases}$

(7) $\sum_{\substack { i=1 \\ \begin{subarray}{c}{i=1 \\ p \mid i+w{ i = 1 \\ \begin{subarray} { c } { i = 1 \\ p | i + w } }\end{subarray}}^{N-1}= \begin{cases}0, & w \in P, \\ 1, & w \notin P .\end{cases}$

(8) $\sum_{\substack{i=0 \\ p l i q q i+w \\ p q t i+w}}^{N-1} 1= \begin{cases}0, & w \in P, \\ 1, & w \notin P .\end{cases}$

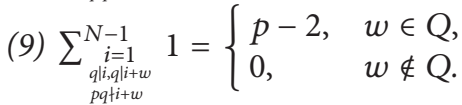

Proof. For the proof of (1) and (6)-(9), one can refer to in [19]; we just prove (2)-(5).

$$
\begin{aligned}
& \sum_{\substack{i=0 \\
\operatorname{gcd}(i, p q)=1 \\
p \mid i+w}}^{N-1} \eta_{i}=\sum_{\substack{i=0 \\
\operatorname{gcd}(i, p q)=1 \\
p \mid i+w}}^{N-1} \eta_{-w}=\eta_{-w} \sum_{\substack{i=0 \\
\operatorname{gcd}(i, p q)=1}}^{N-1} 1= \begin{cases}0, & w \in P, \\
(q-1) \eta_{-w}, & w \notin P,\end{cases} \\
& p \mid i+w \\
& \sum_{\substack{i=0 \\
p \mid i \\
\operatorname{gcd}(i+w, p q)=1}}^{N-1} \eta_{i+w}=\sum_{\substack{i=0 \\
p \mid i \\
\operatorname{gcd}(i+w, p q)=1}}^{N-1} \eta_{w}=\eta_{w} \sum_{\substack{i=0 \\
p \operatorname{li} \\
\operatorname{gcd}(i+w, p q)=1}}^{N-1} 1= \begin{cases}0, & w \in P, \\
(q-1) \eta_{w}, & w \notin P,\end{cases} \\
& \sum_{\substack{i=0 \\
\operatorname{gcd}(i, p q)=1 \\
q \mid i+w, p q t i+w}}^{N-1} \eta_{i}=\sum_{\substack{i=0 \\
\operatorname{gcd}(i, p q)=1 \\
q \mid i+w}}^{N-1} \eta_{i}-\sum_{\substack{i=0 \\
\operatorname{ccd}(i, p q)=1 \\
q|i+w, p q| i+w}}^{N-1} \eta_{i}=0-\sum_{\substack{i=0 \\
\operatorname{gcd}(i, p q)=1 \\
q|i+w, p q| i+w}}^{N-1} \eta_{-w}=-\eta_{-w} \sum_{\substack{i=0 \\
\operatorname{gcd}(i, p q)=1 \\
q|i+w, p q| i+w}}^{N-1} 1= \begin{cases}-\eta_{-w} & w \in Z_{N}^{*} \\
0 & w \notin Z_{N}^{*}\end{cases} \\
& \sum_{\substack{i=1 \\
q \mid i \\
\operatorname{gcd}(i+w, p q)=1}}^{N-1} \eta_{i+w}=\sum_{\substack{i=0 \\
q \mid i \\
\operatorname{gcd}(i+w, p q)=1}}^{N-1} \eta_{i+w}-\sum_{\substack{i=0 \\
q \mid i \\
\operatorname{gcd}(i+w, p q)=1}}^{N-1} \eta_{i+w}=0-\sum_{\substack{i=0 \\
q \mid i \\
\operatorname{gcd}(i+w, p q)=1}}^{N-1} \eta_{w}=-\eta_{w} \sum_{\substack{i=0 \\
q \mid i \\
\operatorname{gcd}(i+w, p q)=1}}^{N-1} 1=\left\{\begin{array}{l}
-\eta_{w} w \in Z_{N}^{*}, \\
0 \quad w \notin Z_{N}^{*} .
\end{array}\right.
\end{aligned}
$$


Theorem 1. Let $S=\left\{s_{i}\right\}$ be the new binary sequences defined in (44); then, the autocorrelation of $S$ is

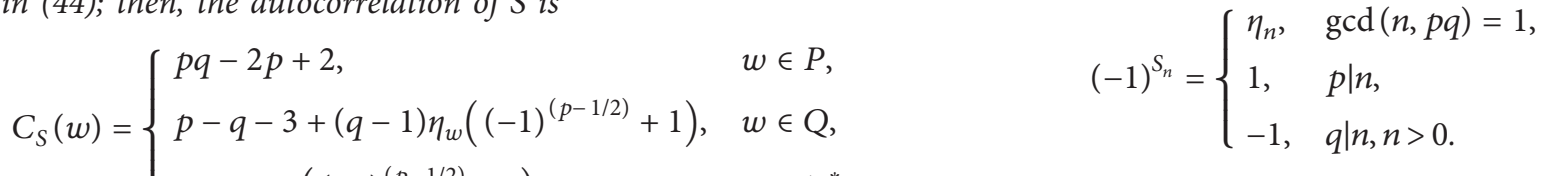

$$
\begin{aligned}
& w \in Z_{N}^{*} . \quad \text { Let } 1 \leq w \leq N-1 \text {; then, }
\end{aligned}
$$

$$
\begin{aligned}
& C_{S}(w)=\sum_{i=0}^{N-1}(-1)^{s_{i+w}+s_{i}}=\sum_{\substack{i=0 \\
\operatorname{gcd}(i, p q)=1 \\
\operatorname{gcd}(i+w, p q)=1}}^{N-1} \eta_{i(i+w)}+\sum_{\substack{i=0 \\
\operatorname{gcd}(i, p q)=1 \\
p \mid i+w}}^{N-1} \eta_{i}-\sum_{\substack{i=0 \\
\operatorname{gcd}(i, p q)=1 \\
q \mid i+w, p q t i+w}}^{N-1} \eta_{i}-\sum_{\substack{i=1 \\
q \operatorname{cod} \\
\operatorname{gcd}(i+w, p q)=1}}^{N-1} \eta_{i+w}
\end{aligned}
$$

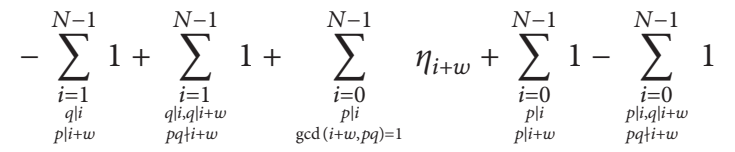

$$
\begin{aligned}
& = \begin{cases}p q-2 p+2 & \text { if } w \in P, \\
p-q-3+(q-1) \eta_{w}\left((-1)^{(p-1 / 2)}+1\right) & \text { if } w \in Q, \\
-q+q \eta_{w}\left((-1)^{(p-1 / 2)}+1\right) & \text { if } w \in Z_{N}^{*} .\end{cases}
\end{aligned}
$$

The autocorrelation function of the new sequences $C_{S}(w)$ is 5-level if $p \equiv 1 \bmod 4 . C_{S}(w)$ is 3-level.

\subsection{Linear Complexity and Minimal Polynomial of Our New} Sequences

Lemma 11 (see [19]). Let $V_{0}$ and $V_{1}$ be the sets defined above; then,

$$
\begin{cases}t V_{0} \in V_{0}, t V_{1} \in V_{1}, & \text { if } t \in V_{0} \\ t V_{0} \in V_{1}, t V_{1} \in V_{0}, & \text { if } t \in V_{1}\end{cases}
$$

Denote

$$
\begin{aligned}
S(x) & =s_{0}+s_{1} x+s_{2} x^{2}+\cdots+s_{N-1} x^{N-1} \\
& =\sum_{i \in C_{1}} x^{i}=\sum_{i \in Q} x^{i}+\sum_{i \in V_{1}} x^{i}
\end{aligned}
$$

Assume $m=\operatorname{ord}_{p q}(2)$ and $\alpha$ is a $p q$ th primitive root of unity in finite field $F_{2^{m}}$. According to the Blahut theorem, the linear complexity of sequence $S=\left\{s_{i}\right\}$ is

$$
\mathrm{LC}(S)=p q-\left|\left\{t: S\left(\alpha^{t}\right)=0, \quad 0 \leq t \leq p q-1\right\}\right| .
$$

Lemma 12. Let $1 \leq t \leq N-1$; then,

$$
S\left(\alpha^{t}\right)= \begin{cases}S(\alpha), & t \in V_{0}, \\ 1+S(\alpha), & t \in V_{1}, \\ \frac{p-1}{2} \bmod 2, & t \in P, \\ 1, & t \in Q, \\ 0, & t \in\{0\} .\end{cases}
$$

Proof. Let $t \in V_{0}$; then, by Lemma 11,

$$
\begin{aligned}
S\left(\alpha^{t}\right) & =\sum_{i \in Q} \alpha^{t i}+\sum_{i \in V_{1}} \alpha^{t i} \\
& =\sum_{i \in t Q} \alpha^{i}+\sum_{i \in t V_{1}} \alpha^{i} \\
& =\sum_{i \in Q} \alpha^{i}+\sum_{i \in V_{1}} \alpha^{i}=S(\alpha) .
\end{aligned}
$$

Let $t \in V_{1}$,

$$
\begin{aligned}
0 & =\alpha^{p q}-1=(\alpha-1)\left(1+\alpha+\cdots+\alpha^{p q-1}\right) \\
& =(\alpha-1)\left(1+\sum_{i \in V_{0}} \alpha^{i}+\sum_{i \in V_{1}} \alpha^{i}+\sum_{i \in P} \alpha^{i}+\sum_{i \in Q} \alpha^{i}\right) .
\end{aligned}
$$


Then, by Lemma 11,

Let $t \in P$; then,

$$
\begin{aligned}
S\left(\alpha^{t}\right) & =\sum_{i \in Q} \alpha^{t i}+\sum_{i \in V_{1}} \alpha^{t i} \\
& =\sum_{i \in t Q} \alpha^{i}+\sum_{i \in t V_{1}} \alpha^{i} \\
& =\sum_{i \in Q} \alpha^{i}+\sum_{i \in V_{0}} \alpha^{i} \\
& =\sum_{i \in Q} \alpha^{i}-\sum_{i \in V_{1}} \alpha^{i}+1 \\
& \equiv(S(\alpha)+1)(\bmod 2) .
\end{aligned}
$$

$$
\begin{aligned}
V_{1} \bmod q & =\left\{g^{s} x^{l} \bmod q: 0 \leq s \leq e-1,0 \leq l \leq 1,2 \nmid s+l\right\} \\
& =\left\{g^{2 s} x \bmod q: 0 \leq s \leq \frac{e}{2}-1\right\} \cup\left\{g^{2 s+1} \bmod q: 0 \leq s \leq \frac{e}{2}-1\right\} \\
& =\left\{g^{2 s} \bmod q: 0 \leq s \leq \frac{e}{2}-1\right\} \cup\left\{g^{2 s+1} \bmod q: 0 \leq s \leq \frac{e}{2}-1\right\}=\{1,2, \ldots, q-1\} .
\end{aligned}
$$

When $s$ runs through $\{0,1, \ldots, e-1\}, l$ runs through $\{0,1\}$, and $2 \nmid s+l$, the set $V_{1} \bmod q$ takes on each element in $\{1,2, \ldots, q-1\}$ exactly $(p-1 / 2)$ times. Therefore,

$$
\begin{aligned}
S\left(\alpha^{t}\right)= & \sum_{i \in Q} \alpha^{t i}+\sum_{i \in V_{1}} \alpha^{t i}=((p-1) \bmod 2)+\left(\frac{p-1}{2} \bmod 2\right) \\
& \cdot \sum_{i \in P} \alpha^{i}=\frac{p-1}{2} \bmod 2 .
\end{aligned}
$$

Let $t \in Q$; then,

$$
\begin{aligned}
V_{1} \bmod p & =\left\{g^{s} x^{l} \bmod p: 0 \leq s \leq e-1,0 \leq l \leq 1,2 \nmid s+l\right\} \\
& =\left\{g^{2 s} x \bmod p: 0 \leq s \leq \frac{e}{2}-1\right\} \\
& \cup\left\{g^{2 s+1} \bmod p: 0 \leq s \leq \frac{e}{2}-1\right\} \\
& =\left\{g^{2 s+1} \bmod p: 0 \leq s \leq \frac{e}{2}-1\right\}=\left\{g^{1}, g^{3}, \ldots, g^{p-2}\right\} .
\end{aligned}
$$

When $s$ runs through $\{0,1, \ldots, e-1\}, l$ runs through $\{0,1\}$, and $2 \nmid s+l$, the set $V_{1} \bmod p$ takes on each element in $\left\{g^{1}, g^{3}, \ldots, g^{p-2}\right\}$ exactly $q-1$ times. Therefore,

$$
\begin{aligned}
S\left(\alpha^{t}\right)= & \sum_{i \in Q} \alpha^{t i}+\sum_{i \in V_{1}} \alpha^{t i}=\sum_{i \in Q} \alpha^{i}+((q-1) \bmod 2) \\
& \cdot \sum_{i \in\left\{g^{1}, g^{3}, \ldots, g^{p-2}\right\}} \alpha^{i}=-1 \equiv 1 \bmod 2 .
\end{aligned}
$$

Let $t \in\{0\}$; then,

$$
\begin{aligned}
S\left(\alpha^{t}\right) & =S(1)=p-1+\frac{(p-1)(q-1)}{2} \\
& =\frac{(p-1)(q+1)}{2} \bmod 2=0 .
\end{aligned}
$$

Lemma 13. Let $V_{0}$ and $V_{1}$ be the sets defined above; then, $2 \in V_{0}$ if and only if $S(\alpha) \in\{0,1\}$.

Proof. Since the characteristic of finite field $F_{2^{m}}$ is 2, $(S(\alpha))^{2}=S\left(\alpha^{2}\right)$.

Let $2 \in V_{0}$; then, $2 V_{i}=V_{i}, i=0,1$.

$$
\begin{aligned}
(S(\alpha))^{2} & =S\left(\alpha^{2}\right)=\sum_{i \in Q} \alpha^{2 i}+\sum_{i \in V_{1}} \alpha^{2 i} \\
& =\sum_{i \in Q} \alpha^{i}+\sum_{i \in V_{1}} \alpha^{i}=S(\alpha) .
\end{aligned}
$$

Therefore, $S(\alpha) \in\{0,1\}$.

Let $2 \in V_{1}$; then, $2 V_{i}=V_{(i+1) \bmod 2}, i=0,1$.

$$
\begin{aligned}
(S(\alpha))^{2} & =S\left(\alpha^{2}\right)=\sum_{i \in Q} \alpha^{2 i}+\sum_{i \in V_{1}} \alpha^{2 i} \\
& =\sum_{i \in Q} \alpha^{i}+\sum_{i \in V_{0}} \alpha^{i}=S(\alpha)+1 .
\end{aligned}
$$

Therefore, $S(\alpha) \notin\{0,1\}$.

Since $2 \notin P \cup Q, 2 \in V_{0}$ if and only if $S(\alpha) \in\{0,1\}$.

Theorem 2. Let $d_{i}(x)=\prod_{j \in V_{i}}\left(x-\alpha^{i}\right), i=0,1$. Then,

(1) When $p \equiv 1 \bmod 8$, 


$$
\begin{aligned}
\mathrm{LC}(S) & =\frac{p q+p-q-1}{2}, \\
m(x) & =\frac{x^{p q}-1}{\left(x^{q}-1\right) d_{i}(x)} .
\end{aligned}
$$

(2) When $p \equiv-1 \bmod 8$,

$$
\begin{aligned}
\mathrm{LC}(S) & =\frac{p q+p+q-3}{2}, \\
m(x) & =\frac{x^{p q}-1}{\left(x^{q}-1\right) d_{i}(x)} .
\end{aligned}
$$

(3) When $p \equiv 3 \bmod 8$,

$$
\begin{aligned}
\mathrm{LC}(S) & =p q-1, \\
m(x) & =\frac{x^{p q}-1}{x-1} .
\end{aligned}
$$

(4) When $p \equiv-3 \bmod 8$,

$$
\begin{aligned}
\mathrm{LC}(S) & =p q-q, \\
m(x) & =\frac{x^{p q}-1}{x^{q}-1} .
\end{aligned}
$$

Proof. Let $\alpha$ be a pqth primitive root of unity in finite field $F_{2^{m}}$. Then,

$$
\begin{aligned}
& x^{p}-1=\prod_{i \in\{0\} \cup Q}\left(x-\alpha^{i}\right), \\
& x^{q}-1=\prod_{i \in\{0\} \cup P}\left(x-\alpha^{i}\right) .
\end{aligned}
$$

Define $d_{i}(x)=\prod_{j \in V_{i}}\left(x-\alpha^{j}\right), i=0,1$. It can be easily seen that

$$
\begin{aligned}
x^{N}-1 & =(x-1)(x-\alpha) \ldots\left(x-\alpha^{N-1}\right) \\
& =\frac{\left(x^{p}-1\right)\left(x^{q}-1\right) d_{0}(x) d_{1}(x)}{x-1} .
\end{aligned}
$$

Case 1. $p \equiv 1 \bmod 8:$ choose $\alpha$ such that $S(\alpha)=0$. Then,

$$
S\left(\alpha^{t}\right)=\left\{\begin{array}{ll}
1, & t \in Q \cup V_{1}, \\
0, & t \in 0 \cup P \cup V_{0} .
\end{array} \quad L C(S)=p q-q-\frac{(p-1)(q-1)}{2}=\frac{p q+p-q-1}{2}, m(x)=\frac{x^{p q}-1}{\left(x^{q}-1 d_{0} x\right)}\right.
$$

Case 2. $p \equiv-1 \bmod 8$ : choose $\alpha$ such that $S(\alpha)=0$. Then,

$$
S\left(\alpha^{t}\right)=\left\{\begin{array}{ll}
1, & t \in P \cup Q \cup V_{1}, \\
0, & t \in\{0\} \cup V_{0},
\end{array} \quad \mathrm{LC}(S)=p q-1-\frac{(p-1)(q-1)}{2}=\frac{p q+p+q-3}{2}, m(x)=\frac{x^{p q}-1}{(x-1) d_{0}(x)} .\right.
$$

Case 3. $p \equiv 3 \bmod 8$. Then,

$$
\begin{aligned}
& S\left(\alpha^{t}\right)\left\{\begin{array}{l}
\neq 0, \quad t \in P \cup Q \cup V_{0} \cup V_{1}, \\
=0, \quad t \in\{0\},
\end{array}\right. \\
& \operatorname{LC}(S)=p q-1, \\
& m(x)=\frac{x^{p q}-1}{x-1} .
\end{aligned}
$$

Case 4. $p \equiv-3 \bmod 8$. Then,

$$
\begin{aligned}
& S\left(\alpha^{t}\right)\left\{\begin{array}{l}
\neq 0, \quad t \in Q \cup V_{0} \cup V_{1}, \\
=0, \quad t \in\{0\} \cup P,
\end{array}\right. \\
& \operatorname{LC}(S)=p q-q, \\
& m(x)=\frac{x^{p q}-1}{x^{q}-1} .
\end{aligned}
$$

The linear complexity of the new sequences is $\mathrm{LC}(S)>(p q / 2)$ if $p \equiv 1 \bmod 8, p>q+1$, or $p \equiv-1 \bmod 8$; $\mathrm{LC}(S)=p q-q$ or $p q-1$ if $p \equiv \pm 3 \bmod 8$, which is very close to period $p q$.

The following are some examples.

Example 1. Let $p=7$ and $q=3$. Then,

$$
\begin{aligned}
P & =\{7,14\}, \\
Q & =\{3,6,9,12,15,18\}, \\
V_{0} & =\{1,2,4,8,11,16\}, \\
V_{1} & =\{5,10,13,17,19,20\} .
\end{aligned}
$$

Our corresponding new binary sequence of period 21 is as follows: 000101100110110101111 .

By using Magma, the autocorrelation value of the above sequence is 3-level, which is consistent with the case $p \equiv 3 \bmod 4$ in Theorem 1 . And the linear complexity of the 
TABle 1: Comparison of autocorrelation $C_{S}(w)$.

\begin{tabular}{lcc}
\hline$C_{S}(w)$ & Sequences of Liu and Chen & New sequences \\
\hline$w \in P$ & $p q-2 p-2$ & $p q-2 p+2$ \\
$w \in Q$ & $1+p-q-(q-1) \eta_{w}\left((-1)^{(p-1 / 2)}+1\right)$ & $p-q-3+(q-1) \eta_{w}\left((-1)^{(p-1 / 2)}+1\right)$ \\
$w \in Z_{N}^{*}$ & $-q-(q-2) \eta_{w}\left((-1)^{(p-1 / 2)}+1\right)$ & $-q+q \eta_{w}\left((-1)^{(p-1 / 2)}+1\right)$ \\
\hline
\end{tabular}

TABLE 2: Comparison of linear complexity LC $(S)$.

\begin{tabular}{lcc}
\hline LC $(S)$ & Sequences of Liu and Chen & New sequences \\
\hline$p \equiv 1 \bmod 8$ & $(p q-p+q-1) / 2$ & $(p q+p-q-1) / 2$ \\
$p \equiv-1 \bmod 8$ & $((p-1)(q-1)) / 2$ & $(p q+p+q-3) / 2$ \\
$p \equiv 3 \bmod 8$ & $p q-p-q+1$ & $p q-1$ \\
$p \equiv-3 \bmod 8$ & $p q-p$ & $p q-q$ \\
\hline
\end{tabular}

above sequence is equal to 14 , which is consistent with the case $p \equiv-1 \bmod 8$ in Theorem 2 .

Example 2. Let $p=11$ and $q=3$. Then,

$$
\begin{aligned}
P & =\{11,22\}, \\
Q & =\{3,6,9,12,15,18,21,24,27,30\}, \\
V_{0} & =\{1,4,5,14,16,20,23,25,26,31\}, \\
V_{1} & =\{2,7,8,10,13,17,19,28,29,32\} .
\end{aligned}
$$

Our corresponding new binary sequence of period 33 is as follows: 001100111110110101110100100111101.

By using Magma, the autocorrelation value of the above sequence is 3-level, which is consistent with the case $p \equiv 3 \bmod 4$ in Theorem 1 . And the linear complexity of the above sequence is equal to 32 , which is consistent with the case $p \equiv 3 \bmod 8$ in Theorem 2 .

Example 3. Let $p=13$ and $q=3$. Then,

$$
\begin{aligned}
P & =\{13,26\}, \\
Q & =\{3,6,9,12,15,18,21,24,27,30,33,36\}, \\
V_{0} & =\{1,4,10,14,16,17,22,23,25,29,35,38\}, \\
V_{1} & =\{2,5,7,8,11,19,20,28,31,32,34,37\} .
\end{aligned}
$$

Our corresponding new binary sequence of period 39 is as follows: 001101111101100100111100100110111110110.

By using Magma, the autocorrelation value of the above sequence is 5-level, which is consistent with the case $p \equiv 1 \bmod 4$ in Theorem 1 . And the linear complexity of the above sequence is equal to 36 , which is consistent with the case $p \equiv-3 \bmod 8$ in Theorem 2 .

Example 4. Let $p=17$ and $q=3$. Then,

$$
\begin{aligned}
P & =\{17,34\}, \\
Q & =\{3,6,9,12,15,18,21,24,27,30,33,36,39,42,45,48\}, \\
V_{0} & =\{1,2,4,8,13,16,19,25,26,32,35,38,43,47,49,50\}, \\
V_{1} & =\{5,7,10,11,14,20,22,23,28,29,31,37,40,41,44,46\} .
\end{aligned}
$$

Our corresponding new binary sequence of period 51 is as follows: 00010111011110110010111110011111010011011 1101110100.

By using Magma, the linear complexity of the above sequence is equal to 32 , which is consistent with the case $p \equiv 1 \bmod 8$ in Theorem 1 , but the autocorrelation value of the above sequence is 4 -level, which is consistent with the case $p \equiv 1 \bmod 4$ in Theorem 2 .

3.3. Comparisons of Results. The comparisons of our results with [19] are listed in Tables 1 and 2.

The comparisons show the following:

(i) When $p \equiv 3 \bmod 4$, the autocorrelation $C_{S}(w)$ of the two sequences is unequal, but they are 3-level. When $p \equiv 1 \bmod 4$, the autocorrelation of the two sequences is unequal, but both of them are 5-level.

(ii) When $p \equiv 1 \bmod 4, p>q$, or $p \equiv 3 \bmod 4$, the linear complexity of our new sequences is larger.

\section{Conclusion}

This paper presents a construction of generalized cyclotomic binary sequences with period $p q$ based on Ding's new cyclotomic class $\left(V_{0}, V_{1}\right)$. And the autocorrelation value, linear complexity, and minimal polynomial of our new sequences are determined. The autocorrelation function $C_{S}(w)$ is 5-level if $p \equiv 1 \bmod 4 . C_{S}(w)$ is 3-level, and $S$ has almost optimal autocorrelation if $p \equiv 3 \bmod 4$. The linear complexity $\operatorname{LC}(S)>(p q / 2)$ if $p \equiv 1 \bmod 8, p>q+1$, or $p \equiv-1 \bmod 8 ; \operatorname{LC}(S)=p q-q$ or $p q-1$ if $p \equiv \pm 3 \bmod 8$, which is very close to the period. The results show that our 
new sequences have quite good cryptographic properties in the aspect of autocorrelation and linear complexity.

\section{Data Availability}

All the data used to support the findings of this study are included in Section 3.2 of this article.

\section{Conflicts of Interest}

The authors declare that they have no conflicts of interest.

\section{Acknowledgments}

This work was supported by the National Natural Science Foundation of China (no. 61902304) and the National Statistical Science Project (2019LY36).

\section{References}

[1] C. F. Gauss, Disquisitiones Arithmaticae, Britannica, Leipzig, Germay, 1801.

[2] A. L. Whiteman, "A family of difference sets," Illinois Journal of Mathematics, vol. 6, no. 2, pp. 107-121, 1962.

[3] C. Ding and T. Helleseth, "New generalized cyclotomy and its applications," Finite Fields and their Applications, vol. 4, no. 2, pp. 140-166, 1998.

[4] I. Damgaard, "On the randomness of Legendre and Jacobi sequences," in Advances in Cryptology; CRYPTO'88, LNCS 403, Springer-Verlag, Berlin, Germany, 1990.

[5] C. Ding, T. Helleseth, and W. Shan, "On the linear complexity of Legendre sequences," IEEE Transactions on Information Theory, vol. 44, no. 3, pp. 1276-1278, 1998.

[6] J. H. Kim and H. Y. Song, "On the linear complexity of Hall's sextic residue sequences," IEEE Transactions on Information Theory, vol. 47, no. 5, pp. 2094-2096, 2001.

[7] C. Ding, "Linear complexity of generalized cyclotomic binary sequences of order 2," Finite Fields and their Applications, vol. 3, no. 2, pp. 159-174, 1997.

[8] C. Ding, "Autocorrelation values of generalized cyclotomic sequences of order two," IEEE Transactions on Information Theory, vol. 44, no. 5, pp. 1699-1702, 1998.

[9] E. Bai, Study on Construction and Randomness Analysis of Pseudorandom Sequences, Xidian University, Xian, China, 2004.

[10] T. Yan, X. Du, G. Xiao, and X. Huang, "Linear complexity of binary Whiteman generalized cyclotomic sequences of order 2k," Information Sciences, vol. 179, no. 7, pp. 1019-1023, 2009.

[11] E. Bai, X. Liu, and G. Xiao, "Linear complexity of new generalized cyclotomic sequences of order two of Length $p q$," IEEE Transactions on Information Theory, vol. 51, no. 5, pp. 1849-1853, 2005.

[12] T. Yan, S. Li, and G. Xiao, "On the linear complexity of generalized cyclotomic sequences of length $p^{m}$," Applied Mathematics Letters, vol. 221, no. 2, pp. 187-193, 2008.

[13] V. Edemskiy, "About computation of the linear complexity of generalized cyclotomic sequences with period $p^{n+1}$," Designs, Codes and Cryptography, vol. 61, pp. 251-260, 2011.

[14] J. Zhang, C.-A. Zhao, and X. Ma, "Linear complexity of generalized cyclotomic binary sequences of length $2 p^{m}$," Applicable Algebra in Engineering, Communication and Computing, vol. 21, no. 2, pp. 93-108, 2010.
[15] L. Hu, Q. Yue, and M. Wang, "The linear complexity of Whiteman's generalized cyclotomic sequences of period $p^{m+1} q^{n+1}$," IEEE Transactions on Information Theory, vol. 58, no. 8, pp. 5534-5543, 2012.

[16] P. Ke, J. Zhang, and S. Zhang, "On the linear complexity and the autocorrelation of generalized cyclotomic binary sequences of length $2 p^{m}$," Designs, Codes and Cryptography, vol. 67, no. 3, pp. 325-339, 2013.

[17] Z. Chang, Y. Zhou, and P. Ke, "Linear complexity of new generalized cyclotomic sequences of order two and length pqr," Acta Electronica Sinica, vol. 43, no. 1, p. 166, 2015.

[18] C. Ding, "Cyclotomic constructions of cyclic codes with length being the product of two primes," IEEE Transactions on Information Theory, vol. 58, no. 4, pp. 2231-2236, 2011.

[19] H. Liu and X. Chen, "Autocorrelation values and linear complexity of new generalized cyclotimic sequences," Acta Mathematica Sinica (Chinese Series), vol. 62, no. 2, pp. 233246, 2019.

[20] D. Pei, A. Salomaa, and C. Ding, Chinese Remainder Theorem: Applications in Computing, Coding, Cryptography, World Scientific, Singapore, Asia, 1996. 